\title{
FORMACIÓN UNIVERSITARIA E INSERCIÓN LABORAL. LA INQUIETUD POR LA EMPLEABILIDAD
}

\author{
University training and job placement. The concern for employability
}

\author{
María Gloria Paredes, Dra. C. \\ Universidad Nacional de Asunción, Paraguay \\ https://orcid.org/0000-0002-3679-2703 \\ mariagloriaparedes@gmail.com
}

\author{
Luis Ortiz, Dr. C. \\ Escuela de Altos Estudios en Ciencias \\ Sociales, Paris \\ https://orcid.org/0000-0002-1902-8834 \\ lortiz@icso.org.py
}

\begin{abstract}
Palabras claves: Empleabilidad, Educación Superior, Egresados Universitarios, Mercado Laboral, Competencia, UNA
\end{abstract}

Keywords: Employability, Higher Education, University Graduates, Labor Market, Competency, UNA
Recibido: 05 de junio de 2019

Aceptado: 02 de julio de 2019

\section{RESUMEN}

Este estudio ha abordado la empleabilidad de egresados universitarios de la Universidad Nacional de Asunción, definida como el nivel de ajuste de la formación recibida con la ocupación de los profesionales que concluyeron sus estudios y obtuvieron su diploma universitario. Específicamente, se definió el perfil socioeconómico de los egresados de dicho establecimiento de educación superior, las características socioocupacionales y las exigencias demandadas por los empleadores en el mercado de trabajo. A partir de una encuesta con muestra aleatoria aplicada a egresados, así como una estrategia cualitativa de entrevista a empleadores los resultados indican una leve mayoría proporcional de egresados empleables, es decir accede a un empleo de la rama de actividad correspondiente a la carrera de egreso, accede al puesto en menos de seis meses después del egreso y se mantiene en el puesto al menos un año. En cualquier sector económico el mercado laboral requiere profesionales con sólida formación académica, que además requiere contar con experiencia profesional en el área de desempeño, es decir las habilidades y destrezas propias de la profesión. Finalmente, la demanda laboral antepone cada vez con mayor fuerza un criterio de contratación consistente en un conjunto de competencias tanto ambiguas como arbitrarias y discrecionales que se anidan bajo el término de "competencias blandas". La empleabilidad de los egresados, a través de una oferta con perfiles académicos que satisfacen los requerimientos demandados, no se debe a la universidad porque se trata de facultades que ésta no puede ni está previsto que provea.

\section{ABSTRACT}

This study has addressed the employability of university graduates of National University of Asuncion, defined as the level of adjustment of the training received with the occupation of professionals who completed their studies and obtained their university degree. Specifically, the socioeconomic profile of the graduates, the socio-occupational characteristics and the demands demanded by employers in the labor market were defined. From a random sample survey applied to graduates, as well as a qualitative interview strategy to employers, the results indicate a slight proportional majority of employable graduates, that is, access to a job in the branch of activity corresponding to the graduation career, Access to the position in less than six months after graduation and stay in the position for at least one year. In any economic sector the labor market requires professionals with solid academic training, which also requires professional experience in the area of performance, that is, the skills and abilities of the profession. Finally, labor demand puts more and more force a hiring criterion consisting of a set of ambiguous as well as arbitrary and discretional competences that are nested under the term "soft skills". The employability of graduates, through an offer with academic profiles that meet the requirements demanded, is not due to the university because it is faculties that it cannot and is not expected to provide. 


\section{INTRODUCCIÓN}

La empleabilidad es un constructo complejo que surge con un significado casi literal de la palabra inglesa: habilidad para obtener o conservar un empleo y, para evitar el desempleo (Campos, 2003). En su evolución se le dotó de más contenido: capacidad o aptitud de una persona para tener un empleo que satisfaga sus necesidades profesionales, económicas, así como de promoción y desarrollo a lo largo de su vida (Martínez, 2011). Lo que implicaba a su vez considerar otras modalidades de trabajo, entre otras cosas formación continua, diseño de un plan de carrera, etc., que en esta acepción supone un acercamiento al concepto de competencia y una mayor riqueza de contenido respecto a lo que significa e implica conseguir un empleo.

La empleabilidad entendida como el nivel de ajuste de la formación recibida en la universidad con el empleo efectivo obtenido por los egresados universitarios es la definición que adoptamos en este estudio. Dicho ajuste tiene lugar cuando el individuo accede a un empleo vinculado directamente al campo profesional de su formación (pertinencia disciplinaria), obtiene el empleo dentro del lapso de 6 (seis) meses de haber egresado de su carrera universitaria (oportunidad de inserción) y, además, conserva dicho empleo por más de un año de modo continuo (permanencia laboral). En función a esta definición, se analizarán los factores que influyen en la empleabilidad.

La bibliografía sobre la temática refiere factores individuales y sociales. Así, por ejemplo, los hallazgos de las investigaciones de Rentería (2006), como los de Álvarez, Bustos y Valencia (2004) coinciden en dar mayor importancia a ciertos factores individuales asociados a la empleabilidad (específicamente la iniciativa, la flexibilidad, la adaptación y la capacidad de interacción), que a otros factores sociales (origen social, etnia, género, credo, apertura y cierre de establecimientos económicos, ampliación o reducción de puestos, aumentos o recortes salariales, entre otros).

El tema de investigación es relevante considerando que la preocupación por la empleabilidad, especialmente de los jóvenes, está en todas las agendas políticas de la actualidad. El presente estudio aborda la empleabilidad de manera crítica. Se enfoca en las experiencias académicas y laborales de egresados universitarios entrelazadas con el rendimiento estudiantil y la eficacia interna de las instituciones encargadas de la formación profesional de universitarios dentro del proceso de evaluación institucional y acreditación de las carreras. Asimismo, se propone identificar los determinantes de la vinculación entre educación superior y empleo proveyendo información sobre el efecto de la enseñanza en el acceso, permanencia y promoción en un empleo o bien si hay otros factores que impactan en la salida laboral de los egresados universitarios.

De este modo, este estudio propone una explicación de la incidencia de la Universidad Nacional de Asunción (UNA), de Paraguay, en la inserción en el mercado de trabajo, bajo la hipótesis de que la UNA tiene poca incidencia en la empleabilidad de sus egresados con una oferta académica que satisface solo parcialmente la demanda de los sectores económicos de mayor dinamismo del mercado laboral nacional. Los objetivos específicos fueron: i) presentar el perfil socioeconómico de los egresados de la Universidad Nacional de Asunción, ii) describir las características laborales de los egresados, iii) estimar los factores individuales y sociales asociados a la empleabilidad de los egresados de la Universidad Nacional de Asunción y, iv) caracterizar cualitativamente la demanda laboral en función de la empleabilidad de los egresados universitarios.

\section{DESARROLLO}

\section{Fundamentación teórica}

Desde la perspectiva económica, la empleabilidad es un conjunto de factores basados principalmente en la formación, que cumplen las expectativas de los empleadores respecto a la cualificación de sus trabajadores (Moreno Mínguez, 2015). El concepto está estrechamente vinculado con el de capital humano que considera al individuo, en tanto trabajador, como factor que genera valor añadido a la empresa.

En la discusión sobre las características de los recursos humanos, Hanushek y Kimko (2000) consideran inadecuada la teoría del capital humano por el énfasis puesto en la cantidad de escolaridad, dado que "la habilidad cognoscitiva de una población no está estimada correctamente por la magnitud de escolaridad ni por los recursos inherentes a la escuela" (p. 1184). En cambio, Psacharopoulos (1994) planteó que el efecto de las habilidades y sus componentes no exceden una proporción dada de escolaridad, de modo que la medición y la inversión en capital humano con base en las tasas de retorno a la educación es pertinente.

La capacidad de la economía siendo reducida para absorber la totalidad (o la mayoría) de los trabajadores egresados del nivel educativo y del superior, en estrecha asociación con la precariedad de programas de incentivo a la inversión 
en fuentes de empleo para profesionales recientemente egresados, Cerrato Reyes y otros (2017) identifican la centralidad que adquirió el criterio de empleabilidad de los profesionales en la gestión de los recursos humanos, haciendo de los factores personales uno de sus componentes fundamentales, además de las características sociodemográficas, las habilidades y destrezas, la movilidad geográfica y la flexibilidad laboral, todos con el propósito de asegurar el acceso, la permanencia y la promoción en el empleo.

En suma, el desarrollo de capital humano en una sociedad depende de las características de la estructura económica, del mercado de trabajo y del modelo de gestión de la empresa económica. Como refieren Brunet y Böcker, las características estándares de la estructura productiva en una época y una circunscripción de la economía de mercado, ligada a una institucionalidad pública específica del capitalismo del Estado de bienestar, se centran en la relación entre formación y trabajo regulada por el modelo de la cualificación, entendida como un atributo del factor trabajo y cuyo valor se estima por su productividad relativa traducida en el salario. La relación formación-trabajo está, en este modelo, constituida de mediaciones sociales que desembocan en una estructura ocupacional, con escalas salariales y jerarquías sociales (Brunet y Böcker, 2014).

Ante la insuficiencia de la teoría de capital humano para explicar la compleja relación de educación y trabajo, surgen abordajes teóricos alternativos como la teoría dualista del mercado laboral o mercado segmentado. El aspecto fundamental de este marco teórico consiste en definir el mercado de trabajo como una esfera no homogénea sino dividida en cuanto menos dos segmentos. Cada segmento posee estructuras y características propias aun si comparten aspectos semejantes entre sí (Méndez e Irepan, 2004).

En una perspectiva crítica que desarrolla esta línea en la sociología de la educación y el trabajo, Bowles y Gintis plantean que la educación influye en la productividad al mejorar las cualificaciones, pero se halla al servicio del aparato productivo capitalista que básicamente constriñe a la población a insertarse en la división social del trabajo en el que las actividades tareas están claramente parceladas y bajo un fuerte control jerárquico (Bowles y Gintis, 1976). Desde esta perspectiva, se define una pirámide ocupacional en la que cada escalafón exige al individuo la posesión de ciertas características individuales, que el sistema productivo requiere y a la vez valora positivamente.

Esta teoría admite, en consonancia con la teoría del capital humano, que los años de educación del individuo e, incluso, su capacidad, influyen en el status profesional, pero atiende una dimensión fundamental que requiere destacar: el origen socioeconómico. El sistema educativo permite la segmentación de los trabajadores y legitima la desigualdad económica, al proporcionar un mecanismo abierto, objetivo y meritocrático de asignación a los individuos en posiciones ocupacionales desiguales y jerarquizadas. El sistema educativo opera por lo tanto como un mecanismo de socialización que imparte a los individuos las formas y cualidades de comportamiento esperado por las empresas. Lo que se requiere de la mayoría es una actitud de conformismo, sumisión, aceptación del puesto asignado y la falta de control sobre el resultado de la actividad (Rahona, 2008).

En otra crítica al modelo económico capitalista, la teoría del capital cultural plantea que la fuerza laboral opera sobre la base de un recurso adquirido en un proceso de transmisión intergeneracional y que se verifica en las competencias que el sujeto puede asimilar y emplear (Bourdieu y Passeron, 1970). Dicho recurso se define como una condición incorporada en el tiempo pero que responde a atributos de clase social ya que es requerida la pertenencia a un grupo de adscripción que poseyendo dichos atributos los transmiten a sus descendientes como parte del principio de conservación de la posición diferenciada y jerárquica que la condición de clase otorga (Bourdieu, 1986).

El mercado laboral, en estructuras económicas heterogéneas, genera fricciones debido a la no correspondencia entre las credenciales profesionales en la oferta y los requerimientos de cualificación de la demanda. Ante este escenario, que caracterizó a la mayoría de los países latinoamericanos, cundió con fuerza el discurso de la empleabilidad. Este apareció como una fórmula que desplaza el problema de la asimetría entre la oferta y la demanda en los mercados de trabajo para imputar la responsabilidad de la dificultad en el acceso al empleo a la población trabajadora. En efecto, el término operacionaliza a modo de categoría residual, los rasgos empíricos de los empleos estables del periodo preneoliberal y que fueron propios de las condiciones laborales en las economías del estado de bienestar (Ortiz et al. 2019).

La noción retira la atención sobre el empleo y sus condiciones objetivas para colocarla sobre el desempleo. Es, en efecto, una reinvención del desempleo, un nuevo marco de significación de esa condición. Como refiere Serrano, "la atención va a ser dirigida a las implicaciones del cambio operado en el centro de gravedad en el análisis del desempleo, al pasar de ser discutido en términos de 'falta de empleo' al de 'carencia de empleabilidad'" (Serrano, 2000, p. 2). En efecto, el reverso de la empleabilidad es por eso el "emprendedurismo", porque instaura con eficacia ideológica un compromiso y una responsabilidad personal -como si de la nada se adquiriera la condición de propietario capitalistapara enfrentar un proceso desfavorable que es colectivo y cuya causa es estructural. Convertirse en "empleable" y volverse "emprendedor" son dos caras de la misma moneda que opera como instrumento de cambio entre una época 
en la que el riesgo (v.gr. el desempleo) era una variable endógena sobre la que se podía tener control y una época en que se convierte en una variable exógena y requiere un nuevo contexto de significado que reafirma lo que Anthony Giddens (1995) denomina la "seguridad ontológica".

Boltanski y Chiapello referían que lo específico de la época del capitalismo en la nueva fase de acumulación (la crisis del estado de bienestar) es su "espíritu" de compromiso con las relaciones capitalistas disociado de las formas institucionales y resignificado como consigna personal. "(...) la mayor parte de las nuevas modalidades de obtener beneficios y las nuevas profesiones, inventadas a lo largo de los últimos treinta años y que generan hoy una parte importante de los beneficios mundiales, han hecho énfasis en lo que la gestión de recursos humanos denomina la 'implicación del personal'. La calidad del compromiso (...) depende más bien de los argumentos que puedan ser invocados para justificar no sólo los beneficios (...) a título individual, sino también las ventajas colectivas (...). Llamamos espíritu del capitalismo a la ideología que justifica el compromiso con el capitalismo" (Boltanski y Chiapello, 2002).

Cuando la composición de la estructura económica se sostiene sobre ramas de actividad con altos niveles de productividad, pero bajos niveles de absorción de la fuerza laboral (producción agropecuaria intensiva), o bien, en ramas de actividad con bajos niveles de productividad y que absorben elevada cantidad de trabajadores, la capacidad productiva de la población económicamente activa, en su conjunto, tiende a ser reducida (Berry, 2017). Por lo tanto, cuando la demanda laboral es baja y la productividad opera de manera heterogénea, la expansión del acceso a la educación no necesariamente redunda en la expansión de capital humano porque la baja inversión de capital y el limitado alcance de la protección social en la población trabajadora, tienden a segmentar el mercado de trabajo, facilitando el acceso a los mejores puestos y a las mejores retribuciones sólo a un sector social, con altas calificaciones educativas que se insertan en los nichos de mayor productividad, mientras los demás sectores no obtienen o ven estancado el retorno a su inversión educativa (Cortés, 2016).

\section{Metodología}

Este estudio moviliza una estrategia combinada de relevamiento empírico y análisis interpretativo. Por una parte, se implementó una encuesta a egresados de la Universidad Nacional de Asunción mientras que por otra se efectuó una serie de entrevistas semiestructuradas a propietarios o directivos de empresas que acogieron a trabajadores egresados de la UNA. Las unidades de observación fueron los egresados con antigüedad de uno a cuatro años de egreso y empleadores en tanto decisores o directivos en establecimientos del área metropolitana de Asunción.

El universo o población estuvo constituido por los graduados de las diferentes carreras de la casa matriz de la Universidad Nacional de Asunción de entre uno a cuatro años de egreso, que hallan desarrollando actividades laborales remuneradas en Asunción y el departamento Central. Se tomaron datos de una muestra de 258 egresados, obtenida de un marco muestral de la Dirección General de Planificación y Estadística del Rectorado de la UNA, constituido por egresados de las diferentes carreras de la casa matriz en el periodo 2010-2013 y distribuidos según áreas disciplinarias (Tabla 1).

El cuestionario de la encuesta contenía preguntas del tipo cerrado con el objetivo de facilitar la codificación durante el procesamiento, así como el análisis de los datos. Los apartados de la encuesta fueron: datos personales, datos familiares, datos académicos, datos laborales y opinión.

Tabla 1. Distribución de la muestra de egresados de la UNA según áreas de conocimiento de la carrera de egreso

\begin{tabular}{lll}
\hline Áreas de conocimiento & Egresados & $\%$ \\
\hline Educación, humanidades y Artes & 5 & 1,9 \\
Ciencias Sociales, Educación Comercial y Derecho & 95 & 36,8 \\
Ciencias & 26 & 10,1 \\
Ingeniería, Industria y Construcción & 44 & 17,1 \\
Agricultura & 51 & 19,8 \\
Salud y Servicios Sociales & 37 & 14,3 \\
\hline Total & $\mathbf{2 5 8}$ & $\mathbf{1 0 0 , 0}$ \\
\hline
\end{tabular}

Por otra parte, fueron entrevistados en total 40 empleadores. Los mismos fueron seleccionados aplicando el muestreo no probabilístico por cuotas en dos etapas a partir del listado de egresados encuestados. Todos ellos están asentados en el área metropolitana de Asunción. En el proceso de selección, en la etapa inicial, se definió el tipo de organización: 
empresas privadas y entidades públicas y, luego en la segunda etapa se seleccionó por sectores económicos de producción, industrialización y servicio según la actividad desarrollada (Gráfico 1).

Gráfico 1. Distribución de los empleadores entrevistados según sector económico.

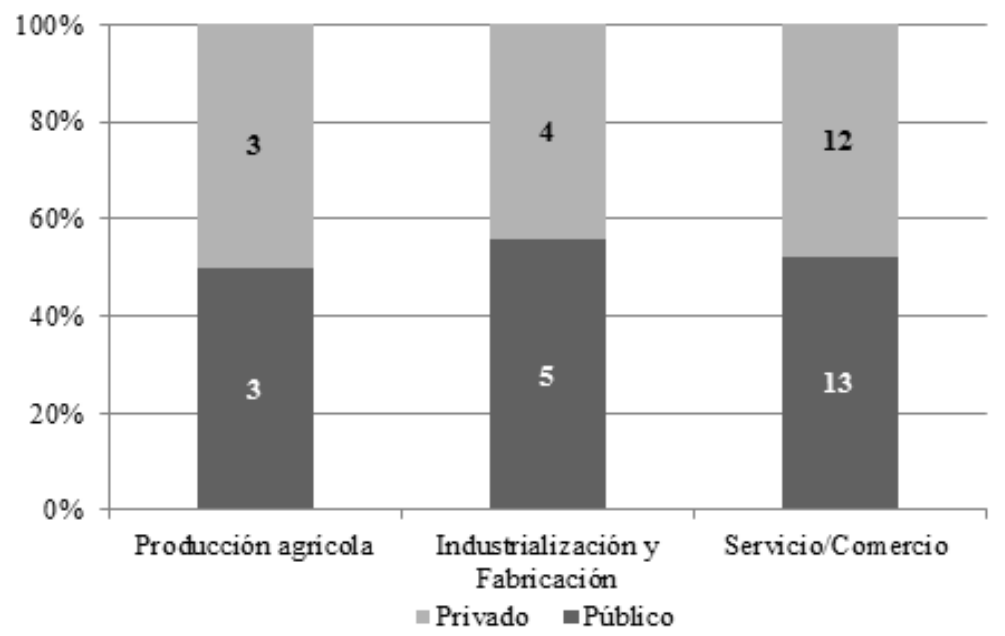

\section{Factores de estudio y variables por dimensión de análisis}

Los egresados fueron encuestados en función a las variables definidas en los factores de estudio, clasificados en individuales y sociales, con sus correspondientes dimensiones (Tabla 2).

Tabla 2. Niveles, dimensiones y variables de estudio de la encuesta a egresados.

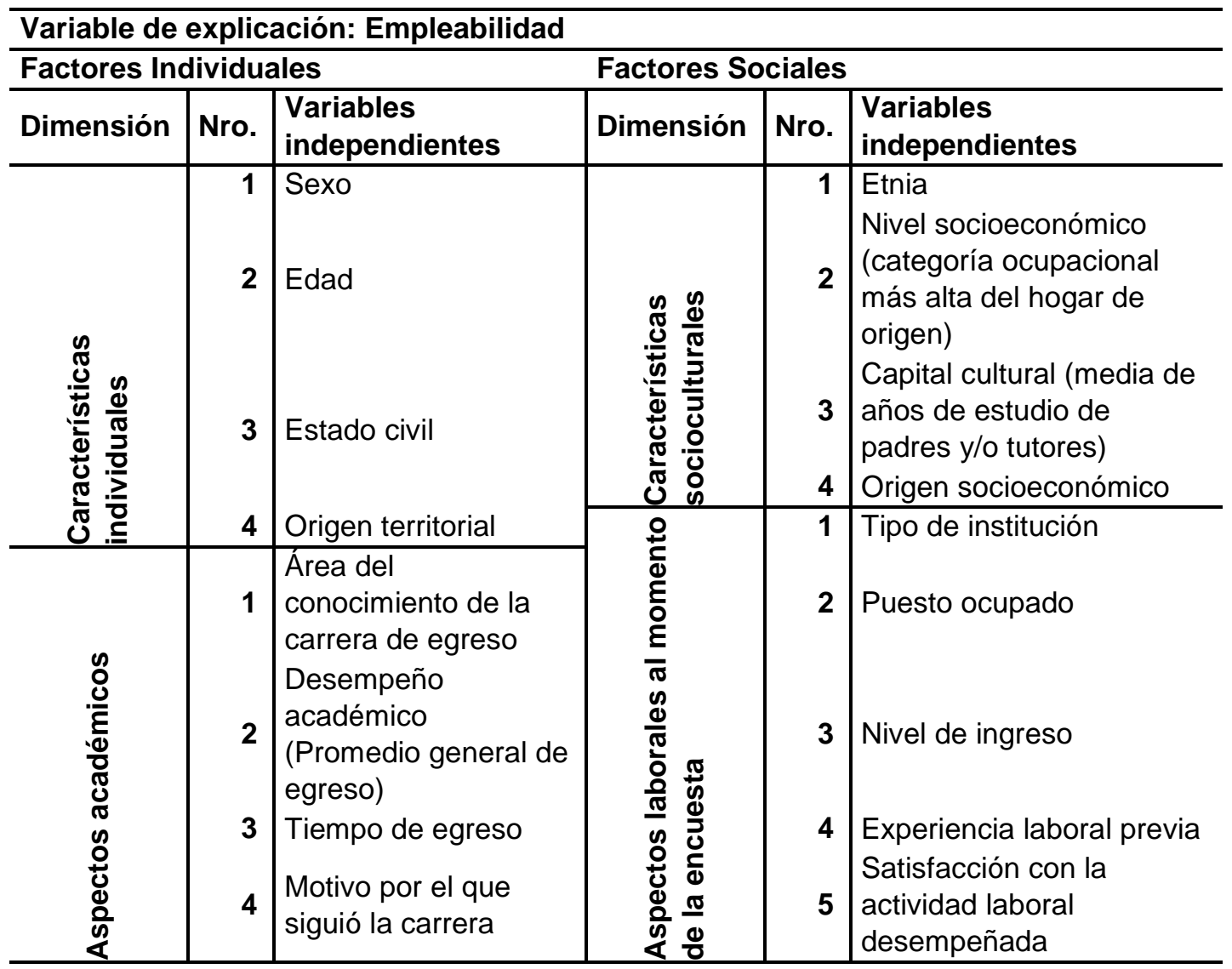

Los empleadores fueron indagados en función a las dimensiones definidas para la descripción del problema estudiado (Tabla 3). La información fue recogida inicialmente por medio de una guía de entrevista semiestructurada de ítems sobre las dimensiones de interés distribuidas en los apartados: características del mercado laboral, aspectos de las competencias de egresados universitarios, demandas de la empresa según el perfil de egreso y los datos generales de la empresa o entidad del empleador entrevistado.

Tabla 3. Dimensiones de análisis e indicadores de la entrevista a los empleadores 


\begin{tabular}{l|l|l}
\hline Dimensión & Descripción & Indicadores \\
\hline $\begin{array}{l}\text { Datos generales de la } \\
\text { empresa }\end{array}$ & $\begin{array}{l}\text { Relevamiento de información } \\
\text { detallada de la empresa o } \\
\text { establecimiento. }\end{array}$ & $\begin{array}{l}\text { Denominación, datos de ubicación y contacto, } \\
\text { tipo de empresa o establecimiento, sector o } \\
\text { actividad económica, número de empleados, } \\
\text { número de empleados universitarios }\end{array}$ \\
\hline $\begin{array}{l}\text { Características del mercado } \\
\text { laboral }\end{array}$ & $\begin{array}{l}\text { Perspectivas del entrevistado } \\
\text { respecto al estado actual del } \\
\text { mercado laboral en su sector de } \\
\text { actividad }\end{array}$ & $\begin{array}{l}\text { Saturación del mercado laboral en el sector de } \\
\text { desempeño }\end{array}$ \\
\hline $\begin{array}{l}\text { Aspectos de las } \\
\text { competencias de egresados } \\
\text { universitarios }\end{array}$ & $\begin{array}{l}\text { Definición de empleabilidad y } \\
\text { descripción de los aspectos } \\
\text { valorados }\end{array}$ & $\begin{array}{l}\text { Competencias, formación académica, } \\
\text { formación práctica, características personales } \\
\text { yamiliares del egresado, otros aspectos de } \\
\text { interés. }\end{array}$ \\
\hline $\begin{array}{l}\text { Valoración del perfil del } \\
\text { egresado de la UNA y del } \\
\text { cumplimiento de la UNA en } \\
\text { la formación de } \\
\text { profesionales con perfiles } \\
\text { adecuados a la demanda. }\end{array}$ & $\begin{array}{l}\text { Valoración del perfil del egresado de de } \\
\text { la UNA y de la formación de } \\
\text { profesionales según sus perfiles. }\end{array}$ & $\begin{array}{l}\text { Descripción del perfil de los egresado de la } \\
\text { UNA y evaluación de la UNA en términos de la } \\
\text { formación de profesionales. }\end{array}$ \\
\hline $\begin{array}{l}\text { Recomendaciones para la } \\
\text { Universidad }\end{array}$ & $\begin{array}{l}\text { Relevamiento de aspectos de } \\
\text { mejoramiento de la formación } \\
\text { universitaria para el desempeño } \\
\text { profesional de los egresados. }\end{array}$ & $\begin{array}{l}\text { Descripción de aspectos académicos, } \\
\text { técnicos, y psicosociales para la adaptación } \\
\text { personal en ambientes de trabajo cambiantes. }\end{array}$ \\
\hline
\end{tabular}

\section{Resultados y discusión}

\section{Los egresados de la UNA y el mercado de trabajo.}

El escenario actual del mercado laboral se halla ante vertiginosos cambios resultantes del proceso de globalización económica, exigiendo un desarrollo productivo fundado en la información y el conocimiento. Este escenario genera en los actores económicos un interés por el desarrollo de las capacidades educativas en la sociedad, demandando al sistema educativo la adecuación de su organización y funcionamiento en respuesta a las demandas y los desafíos de la economía global (Estrada, 2012).

Uno de los principales objetivos que tienen los graduados universitarios al concluir sus estudios es hallar, en el menor lapso posible, un trabajo que les satisfaga y reporte ingresos conforme a sus expectativas (Caballero, López-Miguens y Lampón, 2014.). En el Paraguay, la Universidad busca alcanzar las expectativas de calidad, eficiencia y adecuación de los profesionales egresados a las necesidades laborales y del desarrollo económico. En lo que concierne la oferta universitaria, Paraguay cuenta actualmente con 54 universidades, de las cuales 8 públicas y 46 privadas.

La expansión de las entidades de educación superior ha generado el incremento de la oferta de programas académicos -de grado y posgrado- así como la diversificación profesional en todas las áreas de conocimiento. Entre el 2005 y 2015 se constata una evolución creciente de la tasa de matriculación en la educación superior que impone un mayor esfuerzo en la búsqueda de la eficacia y la calidad educativa. La matrícula ha tenido una explosiva expansión (Tabla 4), pasando de 99.139 estudiantes en 2005 a 320.103 estudiantes en el 2014, alcanzando una tasa media de crecimiento de $14 \%$ anual (CONACYT, 2012).

Tabla 4. Evolución del número estudiantes según tipo de entidad, 2005-2014

\begin{tabular}{|c|c|c|c|c|c|c|c|c|}
\hline \multirow{2}{*}{$\begin{array}{ll}\text { Tipo de } \\
\text { entidad }\end{array}$} & \multicolumn{2}{|l|}{ Año 2005} & \multicolumn{2}{|l|}{ Año 2008} & \multicolumn{2}{|l|}{ Año 2011} & \multicolumn{2}{|l|}{ Año 2014* } \\
\hline & Cantidad & $\%$ & Cantidad & $\%$ & Cantidad & $\%$ & Cantidad & $\%$ \\
\hline Pública & 45.760 & 46 & 58.687 & 34 & 60.013 & 27 & 63.701 & 20 \\
\hline Privada & 53.379 & 54 & 114.395 & 66 & 160.124 & 73 & 256.402 & 80 \\
\hline Total & & 99.139 & 173.082 & & 220.137 & & 320.103 & \\
\hline
\end{tabular}

Fuente: CONACYT, Estadísticas e Indicadores de Ciencia y Tecnología, 2012.

* Dato estimado 
El incremento de la población en edad de acceso a la educación superior y con diplomas de bachillerato, los cambios en las organizaciones y modelos de gestión de las universidades, así como el problema de las posibilidades financieras de solventar la inversión educativa, generó en el periodo en cuestión una superpoblación en la demanda de educación superior incrementándose la cantidad de postulantes y de estudiantes, además de un incremento sustantivo de la cantidad de egresados que deben insertarse en el mercado laboral.

Información estadística de la encuesta permanente de hogares (EPH) del año 2014 respecto al mercado laboral -que concierne a los egresados de la UNA-, refiere que el 19\% de la población económicamente activa (PEA) se halla subocupada y $5 \%$ desocupada (DGEEC, 2015). Del total de los subocupados y desocupados, el 6,4\% tiene 16 o más años de estudio. Las estadísticas oficiales no permiten discriminar con especificidad el porcentaje correspondiente a la población universitaria.

La situación laboral de los egresados de la UNA, según los resultados de la encuesta a los egresados de diferentes carreras1, se caracteriza por el hecho que el $67 \%$ de los que accedieron a un título universitario ya se hallaban trabajando antes de concluir su carrera (UNA, DGPD 2014). En este escenario, se desconoce el nivel de ajuste entre la formación brindada en los establecimientos de educación superior (EES) y las necesidades y demandas del mercado laboral, así como el efecto de la formación universitaria en la trayectoria laboral de los egresados universitarios, problema que fue abordado por este estudio.

En la bibliografía respecto de la relación universidad-empleo, el concepto de empleabilidad de los profesionales egresados aparece como un enfoque de análisis de los factores que inciden en el ajuste entre la formación universitaria y el acceso al empleo según las características y expectativas de la demanda del mercado laboral. A continuación, se presentan los resultados de la encuesta a los egresados y de las entrevistas realizadas a los empleadores.

\section{Perfil socioeconómico de los egresados}

Los resultados de la encuesta indican que la proporción de egresados en condición de "empleabilidad" -según la definición de empleabilidad adoptada- asciende a una proporción del $64 \%$, de la cual el $92 \%$ de los egresados contaba ya con un empleo en el periodo de egreso universitario (Gráfico 2).

Gráfico 2. Distribución de encuestados empleables según condición laboral al egreso.

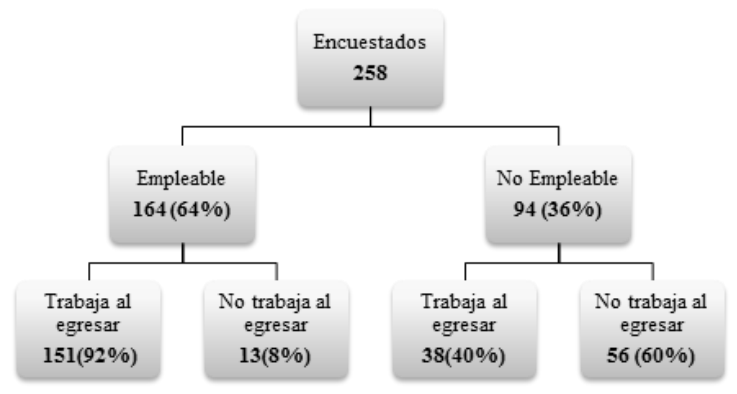

Por otra parte, la encuesta indica que entre los egresados empleables una proporción considerable está constituida por mujeres. Concomitantemente la proporción de mujeres egresadas que no son empleables es superior que la de los hombres en la misma categoría de empleabilidad (Gráfico 3).

Gráfico 3. Distribución de los egresados empleables, según sexo

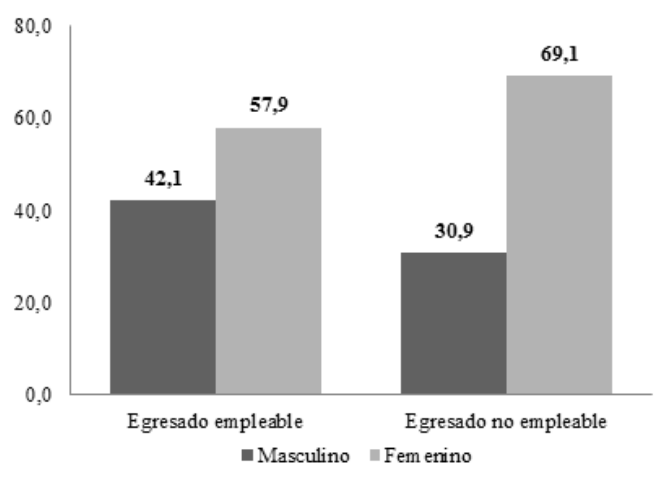

En cuanto a la edad de los egresados empleables, la media es de 26 años mientras que la edad mínima de egreso fue de 22 años. La distribución de los egresados, cuando se agrupa según áreas de conocimiento que corresponden a las 
carreras de egreso (Gráfico 4), se caracteriza por el hecho que la empleabilidad es mayor al $80 \%$ en las carreras del área de Ingeniería, Industria y Construcción, mientras que, en las áreas de Salud y Servicios sociales, así como las de Ciencias sociales, Educación comercial y Derecho, tienen menor porcentaje (65\%). Éstas son seguidas por las áreas de Agricultura (55\%) y Ciencias básicas (46\%), mientras que el área de Educación cuenta con el menor porcentaje de empleabilidad (40\%).

Figura 4. Distribución de los egresados por sexo y empleabilidad

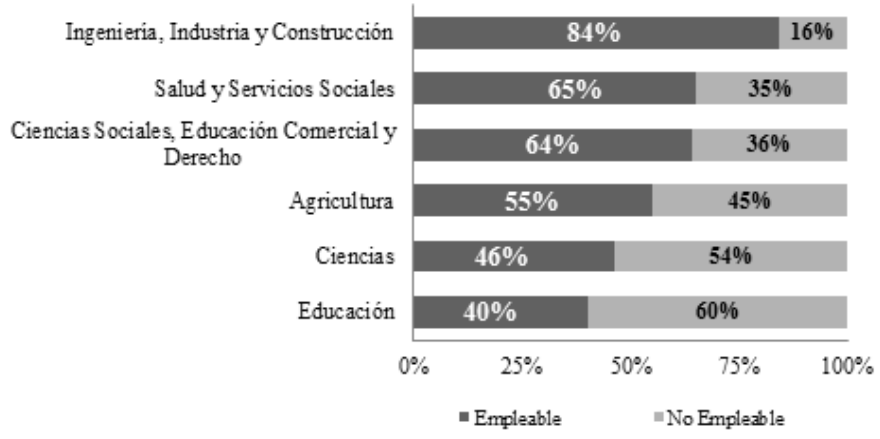

Un aspecto académico que no incide significativamente en la empleabilidad es la calificación académica. Según la distribución de los promedios generales entre los egresados (Gráfico 5), no hay diferencias notorias entre los empleables $(3,79)$ y los no empleables $(3,78)$.

Gráfico 5. Distribución de los egresados empleables por promedio general de egreso
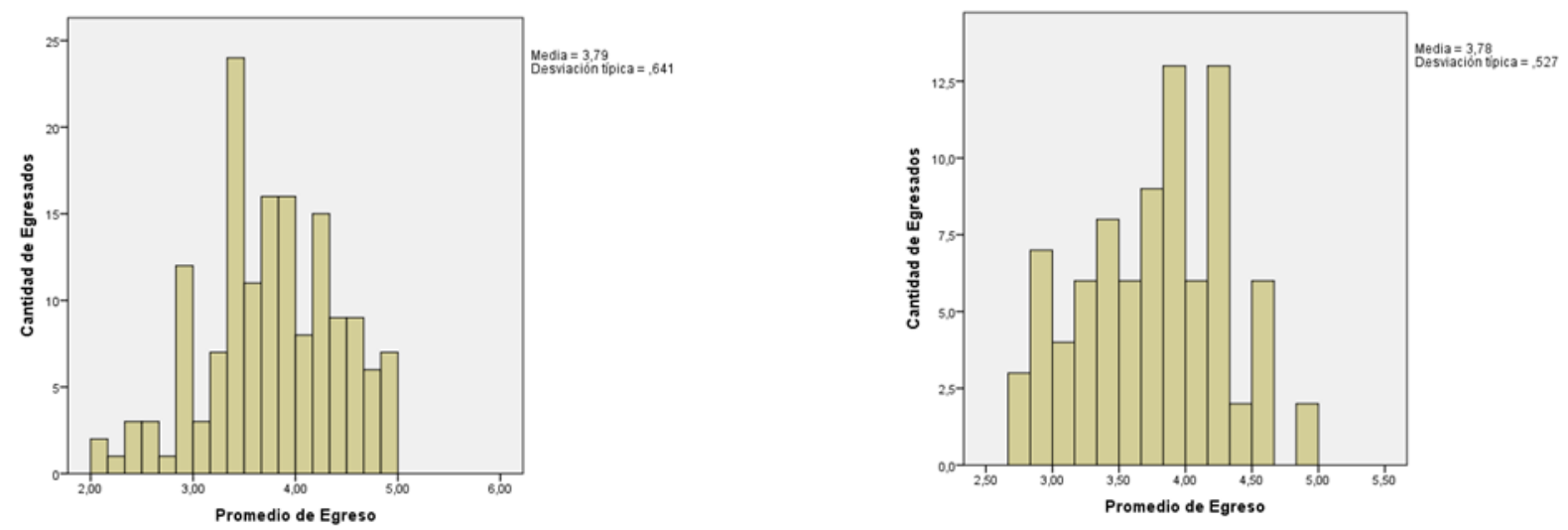

En cuanto al tiempo de egreso, los resultados indican que el $75 \%$ de los egresados empleables obtuvo su titulación universitaria dentro del rango previsto de tiempo de egreso (4 a 7 años), mientras que solo el $67 \%$ de los egresados no empleables culminó en dicho lapso (Tabla 5).

Tabla 5. Egresados por empleabilidad según tiempo de egreso

\begin{tabular}{|c|c|c|c|c|c|c|}
\hline \multirow{2}{*}{ Años } & \multicolumn{2}{|l|}{ Empleable } & \multicolumn{2}{|l|}{ No Empleable } & \multicolumn{2}{|l|}{ Total } \\
\hline & Frec. & $\%$ & Frec. & $\%$ & Frec. & \\
\hline 4 & 8 & 4,9 & 4 & 4,3 & 12 & 4,7 \\
\hline 5 & 33 & 20,1 & 19 & 20,2 & 52 & 20,2 \\
\hline 6 & 56 & 34,1 & 24 & 25,5 & 80 & 31,0 \\
\hline 7 & 25 & 15,2 & 16 & 17,0 & 41 & 15,9 \\
\hline 8 & 7 & 4,3 & 11 & 11,7 & 18 & 7,0 \\
\hline 9 & 6 & 3,7 & 6 & 6,4 & 12 & 4,7 \\
\hline 10 & 8 & 4,9 & 1 & 1,1 & 9 & 3,5 \\
\hline Más de 10 & 21 & 12,8 & 13 & 13,8 & 34 & 13,2 \\
\hline Total & 164 & 100,0 & 94 & 100,0 & 258 & 100,0 \\
\hline
\end{tabular}


En la comparación, entre empleables y no empleables, respecto a los motivos más significativos para estudiar la carrera, los motivos de sugerencia de terceros (66,7\%), la vocación hacia la profesión (67,8\%) así como el aporte a la comunidad de residencia $(66,7 \%)$ constituyen una marcada justificación respecto de los no empleables, mientras que entre éstos sobresale marcadamente la posibilidad de obtener buenos ingresos $(58,8 \%)$ respecto de los egresados empleables (Tabla 6).

Tabla 6. Egresados según motivo por el que eligió la carrera de egreso

\begin{tabular}{|c|c|c|c|c|c|c|}
\hline \multirow{2}{*}{ Motivo } & \multicolumn{2}{|c|}{ Empleable } & \multicolumn{2}{|c|}{ No Empleable } & \multicolumn{2}{|l|}{ Total } \\
\hline & Frec. & & Frec. & & Frec. & $\%$ \\
\hline Influencia familiar & 5 & 55,6 & 4 & 44,4 & 9 & 100,0 \\
\hline Sugerencia de terceros & 6 & 66,7 & 3 & 33,3 & 9 & 100,0 \\
\hline Por Conveniencia & 7 & 63,6 & 4 & 36,4 & 11 & 100,0 \\
\hline Vocación & 61 & 67,8 & 29 & 32,2 & 90 & 100,0 \\
\hline Posibilidad de obtener buenos ingresos & 7 & 41,2 & 10 & 58,8 & 17 & 100,0 \\
\hline Rápida inserción laboral & 7 & 50,0 & 7 & 50,0 & 14 & 100,0 \\
\hline Aporte a la comunidad de residencia & 9 & 60,0 & 6 & 40,0 & 15 & 100,0 \\
\hline Crecimiento y estabilidad económica & 27 & 57,4 & 20 & 42,6 & 47 & 100,0 \\
\hline Por otros motivos & 12 & 92,3 & 1 & 7,7 & 13 & 100,0 \\
\hline
\end{tabular}

Respecto a los factores sociales, en términos del nivel socioeconómico de los egresados entre los empleables y no empleables prevalecen los padres que se desempeñan como trabajadores independientes, seguidos de los trabajadores dependientes y en menor magnitud los trabajadores independientes pero que son docentes universitarios. Con respecto a las madres entre ambos grupos de egresados se verificó que sobresalían aquellas con ocupaciones no remuneradas.

En términos del nivel cultural del hogar de origen (nivel educativo de los padres) es levemente superior la proporción de egresados empleables de nivel cultural bajo, mientras que entre los egresados no empleables ocurre lo contrario: los niveles culturales medio y alto respectivamente son levemente superiores. El dato significativo aquí es que respecto al origen social de los egresados el $60 \%$ de los egresados empleables proviene del nivel bajo mientras que sólo el $6 \%$ del nivel alto (Tabla 7).

Tabla 7. Egresados por empleabilidad según origen social del hogar de origen

\begin{tabular}{lrrrrrrr}
\hline \multirow{2}{*}{ Origen social } & \multicolumn{1}{l}{ Empleable } & \multicolumn{3}{c}{ No Empleable } & \multicolumn{2}{c}{ Total } \\
\cline { 2 - 8 } & Frec. & $\%$ & & Frec. & $\%$ & \multicolumn{1}{c}{ Frec. } & $\%$ \\
\hline Bajo & 100 & 61,0 & 55 & 58,5 & 155 & 60,1 \\
Medio & 54 & 32,9 & 33 & 35,1 & 87 & 33,7 \\
Alto & 10 & 6,1 & 6 & 6,4 & 16 & 6,2 \\
\hline Total & $\mathbf{1 6 4}$ & $\mathbf{1 0 0 , 0}$ & $\mathbf{9 4}$ & $\mathbf{1 0 0 , 0}$ & $\mathbf{2 5 8}$ & $\mathbf{1 0 0 , 0}$ \\
\hline
\end{tabular}

\section{Características laborales de los egresados}

En el estudio fueron también incluidos aspectos laborales. Los egresados fueron consultados sobre su inserción laboral, sobre la situación laboral actual y sobre las perspectivas de desarrollo profesional en el mediano plazo. Respecto a las características del primer empleo los egresados manifestaron desempeñarse en el sector privado mientras que para el segundo empleo mencionaron que se desempeñan generalmente en entidades públicas. Una elevada proporción $(96,3 \%)$ manifestó que la primera ocupación laboral se halla relacionada a la carrera de egreso; una parte considerable relata en los puestos ocupados se da una promoción de auxiliares y técnicos encargados hacia los puestos de supervisores y jefes, aumentando además de dos hasta más de cuatro salarios mínimos. Los egresados manifestaron que su formación universitaria incidió en el acceso al trabajo.

Con respecto al grado de satisfacción con el trabajo actual, el 74,5\% de los egresados manifestó estarlo suficientemente; otra proporción manifestó estarlo mucho, mientras que, entre los no satisfechos, los principales aspectos de disconformidad considerados fueron el nivel de ingreso, el tiempo para efectuar otras actividades laborales y los beneficios sociales (Tabla 8).

Tabla 8. Egresados encuestados según grado de satisfacción en el trabajo 


\begin{tabular}{llllllll}
\hline Aspectos & Nada & Poco & Suficiente & Mucho & $\begin{array}{l}\text { No } \\
\text { responde }\end{array}$ & Total & $\%\left(^{*}\right)$ \\
\hline Nivel de ingreso & 6 & 75 & 134 & 22 & 21 & 258 & 65,8 \\
Desarrollo profesional & 4 & 43 & 124 & 64 & 23 & 258 & 80,0 \\
Perfeccionamiento continuo & 7 & 70 & 96 & 63 & 22 & 258 & 67,4 \\
Facilidad para combinar trabajo y & 6 & 46 & 121 & 63 & 22 & 258 & 78,0 \\
familia & 2 & 27 & 151 & 57 & 21 & 258 & 87,8 \\
Tareas realizadas & 5 & 33 & 119 & 79 & 22 & 258 & 83,9 \\
Ambiente laboral & 10 & 50 & 135 & 42 & 21 & 258 & 74,7 \\
Posición jerárquica & 8 & 40 & 121 & 67 & 22 & 258 & 79,7 \\
Garantía en el Puesto & 22 & 74 & 104 & 36 & 22 & 258 & 59,3 \\
Tiempo para destinar a otras & 29 & 60 & 105 & 43 & 21 & 258 & 62,4 \\
actividades & 5 & 40 & 118 & 71 & 24 & 258 & 80,8 \\
Beneficios sociales & Pertinencia con la formación de egreso & 5 &
\end{tabular}

* Para hallar la proporción de satisfechos se consideró la sumatoria de las categorías Suficiente y Mucho.

Se estimaron los coeficientes de correlación de Spearman $\rho$ (rho) para determinar la relación del nivel salarial y la posibilidad de desarrollo profesional en el nivel de satisfacción con la ocupación desempeñada, obteniéndose que $\rho$ (Satisfacción y Nivel salarial) $=-0,05$ y $\rho$ (Satisfacción y Desarrollo profesional) $=0,023$ (no significativa), es decir estas variables no definen el nivel de satisfacción entre los trabajadores.

La carencia de experiencia fue apuntada por los egresados empleables, así como por los no empleables como la principal dificultad para iniciarse en el empleo. Por otra parte, la falta de práctica fue otra de las dificultades frecuentemente indicadas como obstáculo para iniciarse en el desempeño laboral. Las otras dificultades señaladas son marcadamente de menor frecuencia en comparación a la falta de experiencia (Tabla 9).

Tabla 9. Egresados que trabajan por empleabilidad según dificultad para iniciarse en el empleo

\begin{tabular}{|c|c|c|c|c|c|c|}
\hline \multirow{2}{*}{ Dificultad en el empleo } & \multicolumn{2}{|c|}{ Empleable } & \multicolumn{2}{|c|}{ No Empleable } & \multicolumn{2}{|l|}{ Total } \\
\hline & Frec. & $\%$ & Frec. & $\%$ & Frec. & $\%$ \\
\hline Falta de práctica para resolver problemas & 8 & 11,1 & 14 & 17,7 & 22 & 14,6 \\
\hline Falta de conocimientos actualizados & 6 & 8,3 & 5 & 6,3 & 11 & 7,3 \\
\hline Falta de experiencia & 24 & 33,3 & 29 & 36,7 & 53 & 35,1 \\
\hline Poca demanda de profesionales del área & 8 & 11,1 & 7 & 8,9 & 15 & 9,9 \\
\hline Falta de capital de instalación e iniciación & 4 & 5,6 & 4 & 5,1 & 8 & 5,3 \\
\hline Competencia de otros profesionales & 6 & 8,3 & 6 & 7,6 & 12 & 7,9 \\
\hline $\begin{array}{l}\text { Preferencia por egresados de otras } \\
\text { universidades }\end{array}$ & 1 & 1,4 & - & - & 1 & 0,7 \\
\hline Distancia del hogar al lugar de trabajo & 5 & 6,9 & 4 & 5,1 & 9 & 6 \\
\hline Baja remuneración & 7 & 9,7 & 7 & 8,9 & 14 & 9,3 \\
\hline Otras & 3 & 4,2 & 3 & 3,8 & 6 & 4 \\
\hline Total & 72 & 100,0 & 79 & 100,0 & 151 & 100,0 \\
\hline
\end{tabular}

La proporción de satisfacción de los egresados tanto empleables como no empleables es alta pero la de los empleables es levemente superior que la de los no empleables (81,1\% vs. $77,6 \%)$ (Tabla 10).

Tabla 10. Egresados por empleabilidad según nivel de satisfacción con la formación

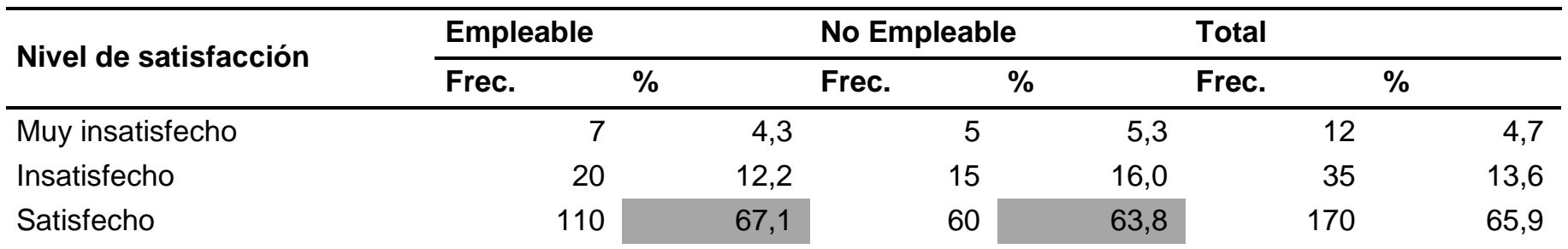




\begin{tabular}{lrrrrrr} 
Muy satisfecho & 23 & 14,0 & 13 & 13,8 & 36 & 14,0 \\
No responde & 4 & 2,4 & 1 & 1,1 & 5 & 1,9 \\
\hline Total & $\mathbf{1 6 4}$ & $\mathbf{1 0 0 , 0}$ & $\mathbf{9 4}$ & $\mathbf{1 0 0 , 0}$ & $\mathbf{2 5 8}$ & $\mathbf{1 0 0 , 0}$ \\
\hline
\end{tabular}

Los aspectos manifestados por los egresados, en los que la universidad requiere poner énfasis difieren en la visión de los empleables y los no empleables. Entre los empleables la universidad debe enfatizar mejoras en la formación académica y científica, estableciendo mecanismos de vinculación entre teoría y práctica. Entre los no empleables la universidad debe mejorar el uso de las TICs y establecer un adecuado vínculo con el entorno a través de la firma de convenios interinstitucionales (Tabla 11).

Tabla 11. Aspectos a enfatizar por la universidad para apuntalar el acceso al empleo.

\begin{tabular}{|c|c|c|c|c|c|c|}
\hline \multirow{2}{*}{ Aspectos } & \multicolumn{2}{|c|}{ Empleable } & \multicolumn{2}{|c|}{ No Empleable } & \multicolumn{2}{|l|}{ Total } \\
\hline & Frec. & $\%$ & Frec. & $\%$ & Frec. & $\%$ \\
\hline Pasantías. & 85 & 63 & 50 & 37 & 135 & 100 \\
\hline Mejorar formación académica & 64 & 72,7 & 24 & 27,3 & 88 & 100 \\
\hline Mejorar formación científica & 53 & 65,4 & 28 & 34,6 & 81 & 100 \\
\hline Idiomas & 62 & 62 & 38 & 38 & 100 & 100 \\
\hline Establecer convenios con empresas & 83 & 57,2 & 62 & 42,8 & 145 & 100 \\
\hline Mejorar la formación práctica & 78 & 64,5 & 43 & 35,5 & 121 & 100 \\
\hline Mejorar el uso de las TICs & 30 & 50 & 30 & 50 & 60 & 100 \\
\hline Movilidad estudiantil y de egresados & 27 & 58,7 & 19 & 41,3 & 46 & 100 \\
\hline Otro & 7 & 77,8 & 2 & 22,2 & 9 & 100 \\
\hline
\end{tabular}

Obs. La pregunta permite marcar hasta dos opciones

Consultados los encuestados respecto a si en su perspectiva perciben que haya demanda para su profesión en el mercado laboral, respondieron afirmativamente el $86,0 \%$ de los egresados empleables y el $74,5 \%$ de los egresados no empleables (Tabla 12).

Tabla 12. Opinión sobre demanda en el mercado laboral actual de acuerdo a profesión de los egresados.

\begin{tabular}{lrrrrrrrr}
\hline \multirow{2}{*}{ Alternativa } & \multicolumn{1}{l}{ Empleable } & \multicolumn{3}{c}{ No Empleable } & \multicolumn{2}{c}{ Total } \\
\cline { 2 - 9 } & Frec. & & $\%$ & & Frec. & $\%$ & Frec. & $\%$ \\
\hline $\mathrm{Si}$ & 141 & 86 & 70 & 74,5 & 211 & 81,8 \\
$\mathrm{No}$ & 17 & 10,4 & 23 & 24,5 & 40 & 15,5 \\
No responde & 6 & 3,7 & 1 & 1,1 & 7 & 2,7 \\
\hline Total & $\mathbf{1 6 4}$ & $\mathbf{1 0 0}$ & $\mathbf{9 4}$ & $\mathbf{1 0 0}$ & $\mathbf{2 5 8}$ & $\mathbf{1 0 0}$ \\
\hline
\end{tabular}

Los aspectos más valorados y manifestados por los encuestados para obtener un empleo y mantenerse en los mismos fueron las competencias demostradas y la formación académica. Se destaca que las características de los hogares de origen constituyen un factor importante en ambas situaciones y que las características subjetivas son importantes para acceder a un puesto, aunque no para mantenerse en el mismo. En la categoría de "Otros" están la disponibilidad de tiempo completo y la posibilidad de residir en el interior, o bien viajar al extranjero, que verifican como importantes en la obtención, así como en la mantención del empleo (Gráfico 6).

Gráfico 6. Distribución de los egresados según valoración de los aspectos indicados para la obtención y mantención del empleo.

\section{Demanda de los empleadores}

Para caracterizar cualitativamente la demanda laboral en términos del reclutamiento de los universitarios egresados con perfiles de empleabilidad, se recurrió a relevar la perspectiva de los empleadores. Se entrevistaron a responsables de contratación (propietarios, rectores, gerentes, directores, jefes de recursos humanos, presidentes de gremios y viceministros) de 40 empresas y/o entidades en las que los egresados encuestados mencionaron hallarse empleados. 
Los perfiles cualitativos demandados por el mercado varían en relación a la rama de actividad. Las esferas del mercado de trabajo en función de los sectores económicos difieren en la duración para la inserción, los requerimientos, la carrera de profesionalización y los procesos de inducción. Las competencias desarrolladas durante las carreras están relacionadas en forma positiva y significativa con las competencias aplicadas en el trabajo (Paredes, 2011), dándose además otras condiciones como ciertas características del egresado (habilidades individuales y destrezas sociales) así como el desarrollo de las habilidades propias del puesto de trabajo que afectan a su condición de empleabilidad.

La información de las entrevistas implementadas indica que, desde la demanda, los empleadores reconocen que la formación académica en la UNA es "buena" pero consideran que otros criterios operan en la selección, mantenimiento y promoción de los empleados además de los conocimientos científicos y técnicos recibidos en la universidad. Estos criterios son la experiencia, las "competencias blandas" y el manejo de aptitudes psicosociales (Tabla 13). Los empleadores manifiestan que se requiere complementar la formación universitaria con conocimientos específicos para el cumplimiento de las funciones en los puestos de contratación. Ahora bien, un motivo clave por el cual utilizan estos criterios de filtro es para controlar los techos salariales de los profesionales contratados, habida cuenta de la propensión a maximizar la ganancia del capital en detrimento de la retribución al trabajo.

Tabla 13. Características de empleabilidad demandadas por los empleadores.

\begin{tabular}{|c|c|}
\hline Aspectos & Descripción \\
\hline Formación académica & $\begin{array}{l}\text { Los pue stos laborales de cua quier sector económico requieren específicamente que el egresado } \\
\text { empleable sea un profe sional que posea: } \\
\text { Sólida formación académica, técnica y man ejo de herramientas informáticas y tecnológicas de } \\
\text { apoyo aplicadas al área (TICs) actualizadas que complementan el trabajo pro fesional } \\
\text { Manejo de un idioma extranjero, el idioma inglés que le da un valor agre gado al profesional dentro } \\
\text { del proceso de internacionalización de la educación superior respecto a investigación y gestión del } \\
\text { conocimiento. } \\
\text { Dominio del idioma guarani sobre todo en algun as áreas que como pro fesionales tienen que } \\
\text { relacionarse con pacientes o usuarios, productores, clientes, alumnos. }\end{array}$ \\
\hline Experiencia laboral & $\begin{array}{l}\text { Experiencia práctica (práctica profesional) en el área a desempeñarse, es decir las habilidades y } \\
\text { de stre zas propias de la profesión (aplicación de la teoria a la práctica). }\end{array}$ \\
\hline \multirow{3}{*}{ Competencias } & $\begin{array}{l}\text { Valoran la experien cia práctica y tener habilidades y destrezas relacionad as a la actividad a } \\
\text { desempeñar en el puesto al que se postula. }\end{array}$ \\
\hline & $\begin{array}{l}\text { Las competencias blandas (habilidades que van más allá de las técnicas de un o ficio y están } \\
\text { vin culadas con la parte emocional, la conducta, las motivaciones, los valores y cultura del ser } \\
\text { humano) para desempeñar con eficien cia y soltura las actividades del puesto en la institución o } \\
\text { empresa. }\end{array}$ \\
\hline & $\begin{array}{l}\text { Las principales competen cias deseadas son: Creatividad, Iniciativa, Proactivo y E spíritu } \\
\text { emprendedor, Aprendizaje autón omo, Independiente, Trabajo bajo presión, Capacitación continua, } \\
\text { Flexibilidad para adaptarse, Liderazgo y Toma de decisiones }\end{array}$ \\
\hline \multirow[b]{2}{*}{ Otras caracteństicas } & La vocación, empatía, capacidad de escuchar, compromiso, sentido de pertenencia, ética y valores \\
\hline & $\begin{array}{l}\text { La actitud positiva (autoestima) en el egresado al momento de solicitar un puesto la boral porque es } \\
\text { un factor determinante para de cidir la contratación de los mismos más que la formación académica. }\end{array}$ \\
\hline
\end{tabular}

En este escenario es posible afirmar que la UNA, en su misión, cumple un papel importante en el desarrollo de las características de "empleabilidad" de los futuros profesionales, pero ¿hasta qué punto la universidad es responsable de dotar de las características de empleabilidad requeridas por el mercado y traducidas a través de las expresiones y demandas de los empleadores?

A partir del perfil profesional demandado por los empleadores que emerge de sus testimonios se infiere que la universidad requiere propiciar un ambiente de experiencias de aprendizaje donde los estudiantes sean los protagonistas. Esto es especialmente importante en la actualidad porque la creatividad y la innovación se aprenden a fuerza de la práctica y en el tiempo. Esta está en correlación con el enfoque de competencias según la cual la enseñanza y el aprendizaje se desarrollan por la acción en situaciones concretas de trabajo.

La valoración de la formación académica y las competencias apoyan la tesis de la teoría del capital cultural, según la cual su incorporación está directamente relacionada a la herencia familiar de cada sujeto y representa lo que, por una parte, su pertenencia de clase condiciona para apropiarse y, por otra parte, lo que el sujeto ha podido asimilar y lo que puede hacer por sí mismo, lo cual se verá incrementado por la inversión en tiempo para el mejoramiento de las formas de aprender (Bourdieu, 1986).

Sin embargo, las "deficiencias" de dotación de ciertos requisitos de "empleabilidad" no son responsabilidad de la Universidad dado que se tratan de criterios extracurriculares que aplican para la gestión de los recursos humanos en el mercado laboral (experiencia, competencias blandas, "inteligencia emocional", entre otros) cuya adopción e incorporación no hacen -ni tienen porqué hacer- parte del proceso académico. Otro aspecto importante a discutir en la formación universitaria de los profesionales que podría impactar de manera importante en la empleabilidad es el 
nivel de generalidad o especificidad de su formación. ¿Son los profesionales generalistas más empleables que los especializados?

La respuesta a esta pregunta desde la perspectiva de los empleadores es que las carreras generalistas afianzan la empleabilidad mientras que los énfasis -especialización- la limitan. Esta visión se refuerza con la idea que el estudiante es empleable desde que ingresa a la universidad, por lo que deducen que la demanda del mercado al momento de acceso a los puestos de trabajo requiere de un profesional medianamente calificado, pero con aptitudes de adaptación a cualquier requerimiento y puesto debido a su formación generalista. Cuando su formación es especializada, sus competencias son específicas y por lo tanto su capacidad de adecuación al entorno laboral más limitada o cuanto menos más difícil de "modear".

Los empleadores consideran que los niveles de postgrado y formación post-título constituyen las instancias de especialización conforme a los requerimientos de la demanda. Considerando la perspectiva empresarial de que la universidad debe formar generalistas, se da el riesgo que ella, como institución de educación superior, se halle bajo la presión de dotar conocimientos específicos, pero concomitantemente debiendo atender la perspectiva generalista de las empresas en la actualidad. En este escenario, no proveerá profesionales ni generalistas ni suficientemente especializados como para dominar una disciplina dada (Vargas, 2001).

Esta discusión implica la reflexión sobre la organización y proyección de la academia en términos de aspectos como la duración de las carreras, la propuesta de énfasis de especialización, la adopción de enfoques prácticos y la oferta de grados intermedios (tecnicaturas) antes que programas de especialización, maestría y doctorado. En este marco, los resultados de este estudio indican que la UNA produce profesionales con condiciones de empleabilidad pero que requiere atender aun aspectos de esta dimensión teniendo en cuenta el énfasis de dicha universidad en la oferta laboral y la formación profesional en carreras de grado con estándares disciplinares rígidos y poca flexibilidad.

En el análisis de la relación del mercado laboral y educación se verifica que un nivel de estudios elevado aumenta la posibilidad de obtener y mantener el puesto de trabajo, reduciendo la tasa de desempleo, así como asegurando un nivel salarial elevado respecto a los trabajadores no titulados, aunque el título no es suficiente. Son además necesarias otras cualidades, habilidades y actitudes, que el mercado demanda de la fuerza laboral en estructura económica caracterizada por altos niveles de subocupación.

\section{CONCLUSIÓN}

El volumen de empleabilidad indica que seis de cada diez egresados logra un ajuste entre la formación recibida en la universidad y el puesto obtenido en su etapa post-universitaria, según la definición adoptada en este estudio. Entre los egresados empleables, predominan las mujeres. La edad media de los egresados empleables es de 26 años.

La distribución de los egresados empleables, según las áreas de conocimiento de las carreras de egreso, se expresa en que la empleabilidad es mayor al $80 \%$ en las carreras del área de Ingeniería, Industria y Construcción, seguida de las áreas de Salud y Servicios sociales y Ciencias sociales, Educación comercial y Derecho tienen menor porcentaje, mientras que el área de Educación cuenta con la proporción más baja de empleabilidad. En los aspectos académicos, entre los grupos estudiados coinciden la media de egreso mientras que la empleabilidad varía por área de conocimiento de la carrera de egreso y motivo por el que emprendió la carrera.

Respecto a los factores sociales, los resultados indican que la proporción de egresados con nivel sociocultural bajo predomina y no se hallan diferencias significativas entre los empleables y no empleables. Además, el capital cultural de los egresados por lo general tanto los empleables como no empleables cuenta con niveles de 12 a 15 años de estudio.

Desde la oferta la UNA tiene poca incidencia en la empleabilidad de sus egresados en el mercado laboral actual. Esto podría deberse a la sobrecarga de oferta por parte de las universidades privadas que lanzan también al mercado profesionales de todas las áreas que compiten con los egresados de la aquella. Desde la demanda, los empleadores reconocen que la formación científica y técnica en la UNA es pertinente, pero consideran otros criterios para la selección, mantenimiento y promoción en los puestos que ofrecen. Estos criterios son la experiencia, las competencias blandas y el manejo de habilidades psicosociales. A partir de esta característica, se percibe que los empleadores exigen una complementariedad entre la formación universitaria y competencias extraídas de la experiencia, así como capacidades de interacción que la universidad no provee.

Sin embargo, una fundamentación crucial por la cual los empleadores utilizarían los criterios subjetivos de filtro es el control de los techos salariales de los profesionales contratados. Esto se debe a que existen grupos ocupacionales 
saturados en el mercado laboral, lo que hace descender la demanda y generar una sobreoferta. Como es de esperar, la media de las retribuciones desciende, de modo que la empleabilidad genera un discurso de desarrollo de capacidades objetivas y subjetivas que permite la adecuación por parte de los profesionales de puestos de remuneración inferior a sus expectativas y que estarían dispuestos aceptar para evitar la desocupación.

Es posible deducir que el campo de actuación de la universidad no corresponde con el desarrollo de la así dicha "empleabilidad" de los egresados dado que entran a regir criterios extracurriculares que no hacen parte ni de los objetivos ni de los programas curriculares ofrecidos por el sistema de educación superior, pero cuya evaluación por parte de los empleadores responde a parámetros en general arbitrarios y discrecionales.

Finalmente, respecto a la hipótesis planteada es posible verificar que la UNA incide parcialmente en la empleabilidad de los egresados, a través de una oferta con perfiles científicos y técnicos que satisfacen los conocimientos teóricos y habilidades prácticas demandados por el mercado laboral aunque la formación de sus egresados en las cualidades utilizadas como filtro en la selección, mantenimiento y promoción de los profesionales, desde la óptica de los empleadores, no son satisfechas por la universidad.

Esta "carencia" de la universidad no responde tanto a un problema de su oferta académica sino a las restricciones que el mercado laboral genera y que la demanda laboral esgrime como argumento para filtrar y seleccionar los egresados "empleables" según criterios extra-académicos y con bajo nivel de previsibilidad, así como de calculabilidad para justificar ideológicamente la "carencia" individual de cada sujeto y asegurar su aquiescencia con un proceso de formación continua y estimulación emocional (dinámicas de "coaching", por ejemplo).

De este modo, la satisfacción parcial de la UNA en la empleabilidad se debe a factores de entorno (mercado laboral) del que la misma tiene poco control, además de que el volumen de la demanda laboral de los sectores de mayor dinamismo del mercado de trabajo nacional no puede ser satisfecho con la baja cantidad de egresados de carreras especializadas en salud, ingenierías y ciencias agrarias, relacionados con dichos sectores (producción agropecuaria y servicios).

Por otra parte, el estudio detectó que la oferta técnica y profesional de las instituciones de educación superior no presentan problemas en la respuesta a la demanda de los empleadores. Es más, en entrevistas a los mismos, muchos expresaban su conformidad e incluso su satisfacción con los niveles de la universidad pública. Los empresarios aggiornados afirman que los aspirantes al empleo no poseen sus mismas características y sus mismas disposiciones, problema -más imaginario que real- que instalan con el discurso eufemístico de la falta de "competencias blandas". Entre éstas, algunas como la "actitud colaborativa", la "autoestima personal", la "aptitud al cambio", entre otras, operan como criterios muy fluctuantes y ambiguos para justificar topes a las retribuciones, transitoriedad de las contrataciones y control sobre los lugares de trabajo.

\section{BIBLIOGRAFÍA}

1. Alvarez A., Bustos D. y Valencia M. (2004). Empleabilidad profesional. Una aproximación a la psicosociología del trabajo. Trabajo de Grado, Instituto de Psicología. Cali: Universidad del Valle.

2. Berry A. (Ed.) (2017), Losing ground in the employment challenge: The case of Paraguay, New York: Routdledged Taylor \& Francis Group.

3. Boltanski L. y Chiapello E. (2002). El nuevo espíritu del capitalismo. Madrid: Akal.

4. Borda D., González C., Ramírez J. y Perera M. (2011). Comportamiento del empleo e ingresos en el Paraguay. Análisis de una década (1997-2008). Asunción: CADEP.

5. Boltanski L. y Chiapello E. (2002). El nuevo espíritu del capitalismo. Madrid: Akal.

6. Borda D., González C., Ramírez J. y Perera M. (2011). Comportamiento del empleo e ingresos en el Paraguay. Análisis de una década (1997-2008). Asunción: CADEP.

7. Bourdieu P. (1986). The forms of capital. In J. Richardson (Ed.) Handbook of Theory and Research for the Sociology of Education. New York: Greenwood.

8. Bowles S. and Gintis H. (1976). Schooling in Capitalist America. Educational Reform and the Contradictions of Economic Life. New York: Basic Books.

9. Brunet I., Böcker Zavaro R. (2014). Competitividad, Competencias y Fin del Ciclo Fordista. International Journal of Sociology of Education, 3(1).

10. Caballero G., López-Miguens M.J. y Lampon J. (2014). La universidad y su implicación con la empleabilidad de sus graduados. Revista Española de Investigaciones Sociológicas (REIS), 146(1), 23-45. 
11. Cáceres E., Gaona M.T., Aguilera Y. y Volpe Rios S. (2005). Educación superior privada en Paraguay. Asunción: IESALC-UNESCO/Universidad Católica Nuestra Señora de la Asunción.

12. Campos Ríos G. (2003). Implicancias Económicas del Concepto de Empleabilidad. Revista de la Facultad de Economía, 7(23).

13. Cañedo Villarreal R. (2010). Educación superior y mercado de trabajo: El caso de los egresados de la Universidad Autónoma de Guerrero. Tesis Doctoral. San José: DUCERE, 206 págs.

14. Cerrato Reyes K., Argueta L., y Zavala J. (2017). Determinantes de la empleabilidad en el mercado laboral. Economía y Administración, 7(1).

15. Cortés F. (2016). Discusiones sobre desigualdad y clases sociales en América Latina en los albores del siglo XXI. En L. Ortiz, Desigualdad y clases sociales. Estudios sobre la estructura social paraguaya. Asunción: CEADUC//CLACSO/ICSO.

16. Dirección General de Estadística, Encuestas y Censos (2013). Resultados de la Encuesta Permanente de Hogares. Fernando de la Mora: DGEEC.

17. Estrada M., y John H. (2012). La formación por competencias y el mundo del trabajo: de la calificación a la empleabilidad. Revista de Salud Pública, 14, 98-111.

18. Giddens A. (1995). La constitución de la sociedad. Bases para una teoría de la estructuración. Buenos Aires: Amorrortu.

19. Hanushek E. and Kimko D. (2000). Schooling, Labor-Force Quality, and the Growth of Nations, The American Economic Review, 90 (5), 1184-1208.

20. Martínez González J. (2011). La empleabilidad: una competencia personal y una responsabilidad social. Contribuciones a las Ciencias Sociales. Recuperado de: www.eumed.net/rev/cccss/15/

21. Mendez E. e Irepan J. (2004). La relación educación-trabajo: los enfoques de capital humano y mercados segmentados. Ethnos Educativo, 11, (30).

22. Ortiz L. (Coord.) (2019). Perfiles educativos y estructura productiva. Credencialismo y cualificación en el mercado de trabajo paraguayo. Asunción: CEADUC/ICSO.

23. Ortiz L. (2016). Desigualdad y clases sociales. Estudios sobre la estructura social paraguaya. Asunción: CEADUC-CLACSO-ICSO.

24. Ortiz L. (2014). La educación escolar en Paraguay. El sistema educativo ante los desafíos de la desigualdad. En Gregosz David, Los desafíos de educación preescolar, básica y media en América Latina. Santiago: KAS/SOPLA.

25. Ortiz L. (2005). Las estructuras sociales del empleo. En Asociación Paraguaya de Estudios de Población, Algunos enfoques sobre el empleo y el capital social. Resultados de investigaciones, Asunción: UNFPA/ADEPO.

26. Paredes M. G. (2015). Empleabilidad de los egresados de la Universidad Nacional de Asunción: factores asociados y exigencias en el trabajo. Revista de Investigación en Ciencias Sociales, 2(4).

27. Paredes M. G. (2011). Evaluación de las diferencias en las demandas de competencias laborales a los egresados de la Universidad Nacional de Asunción. Revista de la FACEN.

28. Pscharopoulos G. (1994). Returns to Investment in Education. A Global Update. World Development. 22.

29. Rahona M. M. (2008). La educación universitaria en España y la inserción laboral de los graduados en la década de los noventa. Un enfoque comparado. En Redchuk M., Empleabilidad. Montevideo: CINTERFOR/OIT.

30. Rentaría E. y Andrade V. (2007). Representaciones y acciones de los estudiantes universitarios frente a la empleabilidad. Psicología desde el Caribe, 20, pp. 130-155.

31. Serrano A. (2000). El concepto de empleabilidad en la estrategia europea de lucha contra el desempleo: una perspectiva crítica, Revista del Ministerio de Trabajo y Asuntos Sociales, 21.

32. Tovar M. G., García, I. y Méndez, A. (2013). Factores asociados a la empleabilidad de los psicólogos en San Luis Potosí. Psicología Científica. 15(12). Recuperado de: http://www.psicologiacientifica.com/factores-empleabilidad-psicologos

33. Universidad Nacional de Asunción (2011). Estudio de Seguimiento a egresados de la UNA, DGPD. Recuperado de www.una.py/estadisticas.

34. Universidad Nacional de Asunción (2014). Resultado de Encuesta a egresados de la UNA 2013, DGPD. Recuperado de www.una.py/estadisticas.

35. Universidad Nacional de Asunción (2015). Anuario estadístico 2014, DGPD. Recuperado de www.una.py/estadisticas.

36. Vargas Zuñiga F. (2001). La formación por competencias. Instrumento para el incremento de la empleabilidad. Montevideo: CINTERFOR/OIT. 